\title{
Information Flow in the Brain: Ordered Sequences of Metastable States
}

\author{
Andrew A. Fingelkurts * and Alexander A. Fingelkurts \\ BM-Science-Brain and Mind Technologies Research Centre, P.O. Box 77, FI-02601 Espoo, Finland; \\ alexander.fingelkurts@bm-science.com \\ * Correspondence: andrew.fingelkurts@bm-science.com; Tel.: +358-95414506
}

Academic Editors: Pedro C. Marijuán, Abir U. Igamberdiev and Lin Bi

Received: 29 December 2016; Accepted: 9 February 2017; Published: 13 February 2017

\begin{abstract}
In this brief overview paper, we analyse information flow in the brain. Although Shannon's information concept, in its pure algebraic form, has made a number of valuable contributions to neuroscience, information dynamics within the brain is not fully captured by its classical description. These additional dynamics consist of self-organisation, interplay of stability/instability, timing of sequential processing, coordination of multiple sequential streams, circular causality between bottom-up and top-down operations, and information creation. Importantly, all of these processes are dynamic, hierarchically nested and correspond to continuous brain state change, even if the external environment remains constant. This is where metastable coordination comes into play. In a metastable regime of brain functioning, as a result of the simultaneous co-existence of tendencies for independence and cooperation, information is continuously created, preserved for some time and then dissipated through the formation of dynamical and nested spatio-temporal coalitions among simple neuronal assemblies and larger coupled conglomerates of them-so-called delocalised operational modules.
\end{abstract}

Keywords: information; self-organisation; circular causality; metastability; operational architectonics; operational modules; EEG; operational synchrony; rapid transitional periods

\section{Introduction}

An issue of crucial importance in the studying and understanding of higher cognitive operations in normal and pathological brain states and eventually the nature of consciousness is the origin and dynamics of information flow in the brain [1-3]. The concept of information as a mathematical framework was first proposed in 1948 by the engineer and mathematician Claude Shannon [4] and was the birth to Information Theory [5]. It was originally intended for the analysis of telecommunication systems, but soon branched out into many other fields [6], including neuroscience [7].

From the very beginning, Shannon's information concept was used [8] to estimate the limits of information transmission capacity in neurons, which then laid the foundation for "Neural Information Flow" theory [9,10]. Further, Attneave [11], Barlow [12] and later Shlens and colleagues [13,14] and Schneidman et al. [15] used the information concept as a mutual constraint in studying the structure and function of the neural system [7]. At the same time, despite these and many other valuable contributions to neuroscience, information dynamics within the brain is not adequately captured by the classical description of information [3]. The non-classical aspects of information dynamics include self-organisation $[1,16]$, the interplay of stability/instability $[17,18]$, timing of sequential processing $[2,19,20]$, coordination of the multiple sequential streams [21,22], circular causality between bottom-up and top-down operations [23-25], and information creation [3]. Importantly, all of these processes are dynamic, hierarchically nested and correspond to continuous change of brain states, even when the external environment remains constant [26-29]. 
According to Tognoli and Kelso [25], “(...) it seems that transmission principles do not scale well upward from simple 'channels' of synaptic interactions to the larger and more complex web of evolved brains. Thus, it is without surprise that the brain betrays an essential communicational etiquette: its parts do not behave in a sequential—one-talks-at-a-time-manner (...). It is also overwhelmingly clear that 'inputs' from the environment do not enter a silent system. Brain parts constantly exchange information about their current and past affairs, and what comes in at a given time works more as a 'perturbation' to an already established ballet, an event that weaves itself within a broader scheme of coordinated brain behaviour rather than the sole commander of all things present" ([25], p. 3). All of these nuances show how difficult it is to keep within the Shannonian spirit when applying the information principle to the brain.

The observations above resonate with an ongoing shift in brain informatics paradigm. Since the brain is an active system that retains the characteristics of a complex, nonlinear system with nonequilibrium dynamics [28], reflected in transient evolution of transient states in the form of discrete frames of activity [30] and phase transitions between micro- and macro-levels [31,32], evidenced by the presence of spontaneous neuronal avalanches [33,34] -it creates or generates information as a result of sequential instabilities. Furthermore, such transient instabilities take place on multiple brain scales (micro-, meso-, and macro-) in a nested hierarchy of multiple coordinative processes, where autonomous tendencies coexist together with the interdependent tendencies [18]. The cornerstone of such coordination dynamics is synergetic self-organization [35], which is described through circular causality of adaptive, informationally meaningful, bidirectional couplings on multiple levels $[25,36]$. If one is to consider this multivariability of brain functioning as a whole, wherein the dynamic self-assembling process neuronal masses engage and disengage over time in the form of transient neuronal assemblies, thus allowing the brain to perceive objects or scenes, manipulate mental images and separate remembered parts of an experience, and finally to bind them all together into a coherent whole, then a new and peculiar principle emerges_metastability of brain functioning [18].

While metastability is a well-established concept in physics, it was first formulated in relation to a neural system by Kelso [37], who framed it within a classical model of coordination dynamics called the extended HKB [38] (where HKB stands for Haken, Kelso and Bunz [39]). Metastability in the brain refers to competition of complementary tendencies of cooperative integration and autonomous fragmentation among multiple distributed neuronal assemblies [1,18,40-44]. The interplay of these two tendencies (autonomy and integration) constitutes the metastable regime of brain functioning [1,18], where local (autonomous) and global (integrated) processes coexist not as antagonists but as a complementary pair [45] at all hierarchically nested levels of the brain functional organisation [23]. Such a metastable regime in the brain arises when "the parts are no longer perfect clones of one another (e.g., as in computational models built from collections of identical neurons) [and] when symmetry is broken and interacting parts are recognized in the diversity of their intrinsic behavior" ([25], p. 3) (see also [21,26,27,46-49]). As a result, information is continuously created, preserved for some time and then dissipated by means of transient spatio-temporal coalitions among multiple neuronal assemblies and their operations associated with the emergence or decay of self-organized operational structures in the brain [23]. Thus, we conclude that ordered sequences of metastable states across multiple spatial and temporal scales constitute information flow in the brain.

No other neurobiological theory is comparable to the Operational Architectonics (OA) theory of brain-mind functioning $[2,18,22-24,41,42]$ in its adherence to metastability as the overall phenomenon $[1,44]$ and concrete versions of it such as a sequence of stable transient states $[3,27,30]$ or transient coordination of autonomous processes [48,49]. The OA theory provides a new and comprehensive framework for outlining information emergence and flow in the brain and mind. In this brief review paper, we will use an informal way of description, leaving the modeling and mathematical aspects as well as computational results out because they are largely still to be devised and to maintain intelligibility for the broad audience of this Special Issue. 


\section{The Operational Architectonics (OA) Theory of Brain-Mind Functioning}

In a series of publications $[2,18,22-24,41,42]$, we have presented a theory of brain OA. This theory states that the simplest mental/cognitive operations that are responsible for qualia or simple computations are presented in the brain in the form of local three-dimensional fields produced by transient functional neuronal assemblies, while the complex operations that are responsible for complex operations, objects, images or thoughts are instantiated by coordinated/synchronized simple operations (temporal coupling of local 3D fields) in the form of so-called operational modules (OM) that may be of varied complexity and life-span. As such, brain OA is presented as a highly structured and dynamic extracellular electric field nested in spatial and temporal domains [23,24] and over a range of frequencies [50], thus forming a particular operational space-time (OST) [23]. This OST exists within brain internal physical space-time (IPST) and the best way to capture it is through the electroencephalogram (EEG) measurement [51,52]. The main property of this OST level of brain organization is that it intervenes between IPST level, where it literally resides, and the experiential/subjective phenomenal space-time (PST) level, to which it is isomorphic [23,41]. Furthermore, the OST level has emergent properties relatively independent from the neurophysiological/neuroanatomical properties of the IPST level; however, it has one-to-one correspondence with the PST level that supervenes on the OST level and is ontologically inseparable from it $[23,24]$.

According to the OA theory and in agreement with the metastability principle, OMs (that are produced by repetitive synchronization of lower-order operations performed by many neuronal assemblies along the cortex, with scale-free, power-law dynamics) are metastable because of intrinsic differences (including semantic) in the activity between neuronal assemblies that constitute every given OM: each neuronal assembly is autonomous by doing its own job, while at the same time still retaining a tendency to be coordinated together within the same $\mathrm{OM}$ in order to execute a given macro-operation $[23,24,53]$. A metastable regime gives tremendous functional advantages for the brain-mind system, for example, speed, flexibility, resilience [21,44] and a tremendous increase in the number of available transient states within and between different levels of a nested functional hierarchy and spanning over several time scales $[18,29,54]$, thus allowing an expression of virtually unlimited diversity of informationally-rich and sophisticated mental and cognitive states [25,53].

Such a metastable mode of brain-mind functioning [23,24,53] introduces hierarchical coupling $[51,55]$ between the brain and mind while simultaneously allowing them to retain their individuality (for a conceptual discussion see [53]). When examined from this perspective, mind, cognition, and behavior, as well as brain activity, are all seen as dynamic processes that rapidly evolve through a series of informationally consistent, spatially and temporally organized, transient coordination states. In each moment of time, these states (of varying complexity) are defined by the selective coordination of local cortical neuronal assemblies that are interacting by virtue of synchrony of their local electromagnetic fields (frames of activity) which are equivalent to functional operations (OST-level) within the large-scale anatomical structure of the cortex (IPST-level) [23,24,41].

In analyzing the OA framework in physical terms, it could be proposed that such nested architectonics of brain-mind operations (presented as OMs of different complexity) can be described in terms of nested energy frames as analyzed within the Dynamic Universe theory [56], where the flow of information is viewed as the flow of energy [57] with abrupt transients (or rapid transitive periods, RTPs [23,24]) between frames of energy [58]. Such RTPs (fast transitions) among the frames are typically associated with a fast memory drop in the dynamics, so that each self-organized single-frame state is often independent from another and the RTPs themselves. This property is mathematically denoted as a renewal condition; then, the sequence of RTPs as a renewal point process [59-61] is typically associated with the ability of the system to trigger a sequence of complex self-organized metastable structures. Why the long-range memory yielded by self-organization is in fact compatible with the memory-resetting properties of renewal events is discussed by Allegrini et al. [62]. In relation to the brain operational architectonics, this means that within the RTP between two consequent 
metastable OMs, there is a biphasic transitive process: in the first brief phase, there is a drastic and abrupt increase in degrees of freedom among participating neuronal assemblies that is accompanied by a sudden increase in entropy, information and dimensionality [24]. This first phase of RTP is followed by a second brief phase with a quick reduction in the degrees of freedom of neuronal assemblies and rapid decrease in entropy, information and dimensionality, indicating the self-organization of a new informational state expressed in the form of a new OM within brain OST [24]. Such a new $\mathrm{OM}$ (new state) presents the new complex operation, phenomenal image or a thought (for a general conceptualization see $[23,24])$.

\section{Empirical Support}

As follows from the previous discussion, the ordered sequences of metastable states likely depend on (1) repertoire (how many types of states exist); (2) probability of occurrence (functional importance of states of a different type); (3) relative incidence of state type changes (how often the state types change); (4) life-span (maintenance of the relative stability in the neurodynamics within a particular time interval); (5) sequence hierarchy (consistent groupings of state types-steady bundle with one another-that comprise more integral blocks of structural organization). If these characteristics consistently change in a non-random fashion (functional relevance) along with the changes in the functional brain states, cognitive tasks and/or different psychopathological changes, then they indeed constitute information flow in the brain. As was already noted, the most adequate way to study sequences of metastable states is through EEG measurement [51,52].

It is well established that local EEG signals have a piecewise stationary structure which could be presented as a sequence of "glued" stationary processes with different probability characteristics (for the reviews see $[2,26,43]$ ). In this context, every quasi-stationary EEG segment (frame) reflects the oscillatory state of the underlying neurodynamical system i.e., transient neuronal assembly $[2,43]$ which signifies a local functional cortical state [63]. In its turn, every EEG oscillatory state is characterized by multiple EEG oscillations where these oscillations are mixed in different proportions depending on the level of vigilance as well as on perceptual, cognitive and mental operations. In this sense, a particular configuration of EEG oscillations (their repertoire and proportions) characterises/reflects a particular type (or class) of neurons' activities. Thus, a local EEG oscillatory state is a steady, transient and self-organised operational unit which has been proposed, based on the experimental research, to present the basic building block of cortical activity [64]. Empirical EEG studies demonstrate that increased functional loading (multistage memory task) causes a statistically significant (a) increase in state type transition between neighboring EEG epochs/frames of the same EEG-signal and (b) decrease of life-span of these states [63]. Perhaps these changes indicate that during increased functional loading, the brain's operations are completed more dynamically and that there exists a transition to a more differential organization in the electromagnetic field [65]. Moreover, not all functional states occur with the same probability; some of them seem to be "preferred" during a particular condition [63]. Psychopharmacological influence, psychopathology, or cognitive load alterations result in the changes of (a) number of oscillatory types of local EEG states; (b) percentage of dominant oscillatory types of local EEG states; (c) transition probability between distinct oscillatory types of functional states; (d) duration of functional states and (e) parameters of the temporal coincidence of the transitions from one functional state type to another registered in different cortex areas [64]. It is important to note that all of these characteristics (repertoire, probability occurrence, relative incidence, life-span and sequence hierarchy) differ significantly from random processes, and are thus functionally relevant, reflecting different real aspects of information flow within the brain.

Evidently, the brain's informational processes dynamic may be reflected in the transformations of a small number of packages of relatively stable patterns within the cortex field oscillatory activity [50]. It is suggested that particular temporal sequences of several EEG patterns appear in consistent groupings (steady bundle with one another) and comprise more integral blocks of local EEG structural organization. The idea that there may exist stable "super-segments/frames" in the individual/local 
EEG (steady combinations of particular segment/frame types as, for example, type " $\mathrm{A}$ " always follows type " $F$ ") was first shown by Jansen [66] and was replicated by other researchers $[63,64,67,68]$.

As a parallel process to sequential dynamics, it has been demonstrated that a set of local bioelectrical (EEG) fields (produced by transient neuronal assemblies that are located in distinct brain areas) can rapidly couple with one another, thus demarcating the establishment of a particular more global or global metastable spatio-temporal OM in the volumetric OST continuum of the brain (see for review [22]). It was shown that the probability that a particular number of cortical areas are recruited into an OM (defined as the temporal RTP coincidences) is governed by power-law statistics [69]. Such dependency is characteristic for non-ergodic systems, thus suggesting, in contrast to a traditional understanding of the brain as an ergodic system [27], that brain functional activity is rather non-ergodic [70,71]. Further, our analysis revealed that such stabilized spatiotemporal OM configurations also have the transient dynamic which is expressed as a series of sudden transitions between OMs [22]. From an information-theory point of view, one may suppose that OMs that cover most or the whole cortex and are long-lasting are not efficient in the healthy brain [72] because context-dependent information transfer is necessarily very dynamic and it would require very quick and flexible reconfiguration of many co-existent OMs. Empirical evidence supports this intuition [22]: it has been shown that the average life-span of OMs is longest for small OMs that are formed by two neuronal assemblies $(\sim 30 \mathrm{~s})$ and shortest for large OMs that span most or the whole cortex ( 100 milliseconds). In this context, the brain operates as a highly dynamic system where large metastable spatial-temporal patterns of stabilized activity (indexed as OMs) formed only for very brief episodes and then quickly dissipated allowing the brain (as a whole) to have more degrees of freedom to form new metastable OMs needed to execute newly immediately-emerged and ever-changing operations of different complexity [22]. This dynamic can be significantly altered during pharmacological influence, neurological or psychiatric pathology or as result of traumatic brain damage when consciousness (including self-awareness) is minimal or lost completely [73-76]. It may also be intentionally altered through mental training such as meditation [77,78].

\section{Conclusions}

Based on the theoretical conceptualizations and empirical data, we propose that information flow within the brain has to be organized in a specific temporal order along a chain of metastable states within and between different levels of a nested functional architecture of the brain. Such dynamic organisation is also isomorphic to the dynamics of phenomenal/subjective experience [53]. Therefore, it is proposed that the ordered sequence of metastable states is a core component of informational flow in such a complex system as the brain-mind system. This perspective seriously considers repetitions of spatial-temporal patterns (indexed as metastable OMs) at all functional levels, thus capturing both dynamic as well as hierarchical complexities of brain activity that are nested within a multi-scale operational architecture [22-24].

Acknowledgments: We would like to thank Carlos Neves (computer science specialist) for programming, technical, and IT support along multiple experiments and Dmitry Skarin for English editing. The authors received no financial support or funding for the research, authorship, and/or publication of this article.

Author Contributions: Both authors contributed equally to this paper.

Conflicts of Interest: The authors declare no conflict of interest.

\section{Abbreviations}

$\begin{array}{ll}\text { EEG } & \text { Electroencephalogram } \\ \text { OA } & \text { Operational architectonics } \\ \text { OM } & \text { Operational module } \\ \text { RTP } & \text { Rapid transitional period } \\ \text { OST } & \text { Operational space-time }\end{array}$




\section{References}

1. Kelso, J.A.S. Dynamic Patterns: The Self-Organization of Brain and Behaviour; The MIT Press: Cambridge, MA, USA, 1995.

2. Fingelkurts, A.A.; Fingelkurts, A.A. Timing in cognition and EEG brain dynamics: Discreteness versus continuity. Cogn. Process. 2006, 7, 135-162. [CrossRef] [PubMed]

3. Rabinovich, M.I.; Afraimovich, V.S.; Bick, C.; Varona, P. Information flow dynamics in the brain. Phys. Life Rev. 2012, 9, 51-73. [CrossRef] [PubMed]

4. Shannon, C. A mathematical theory of communication. Bell Syst. Techn. J. 1948, 27, 379-423. [CrossRef]

5. Yarrow, S.; Challis, E.; Series, P. Fisher and Shannon information in finite neural populations. Neural Comput. 2012, 24, 1740-1780. [CrossRef] [PubMed]

6. Cover, T.M.; Thomas, J.A. Elements of Information Theory, 2nd ed.; Wiley-Interscience: Hoboken, NJ, USA, 2006.

7. Dimitrov, A.G.; Lazar, A.A.; Victor, J.D. Information theory in neuroscience. J. Comput. Neurosci. 2011, 30, 1-5. [CrossRef] [PubMed]

8. MacKay, D.M.; McCulloch, W.S. The limiting information capacity of a neuronal link. Bull. Math. Biophys. 1952, 14, 127-135. [CrossRef]

9. Rapoport, A.; Horvath, W.J. The theoretical channel capacity of a single neuron as determined by various coding systems. Inf. Control 1960, 3, 335-350. [CrossRef]

10. Werner, G.; Mountcastle, V.B. Neural activity in mechanoreceptive cutaneous afferents: Stimulus-response relations, weber functions, and information transmission. J. Neurophysiol. 1965, 28, 359-397. [PubMed]

11. Attneave, F. Some information aspects of visual perception. Psychol. Rev. 1954, 61, 183-193. [CrossRef] [PubMed]

12. Barlow, H.B. Possible Principles Underlying the Transformation of Sensory Messages. In Sensory Communications; Rosenblith, W.A., Ed.; MIT Press: Cambridge, MA, USA, 1961.

13. Shlens, J.; Field, D.G.; Gauthier, L.J.; Grivich, M.I.; Petrusca, D.; Sher, A.; Litke, A.M.; Chichilnisky, E.J. The structure of multi-neuron firing patterns in primate retina. J. Neurosci. 2006, 26, 8254-8266. [CrossRef] [PubMed]

14. Shlens, J.; Field, D.G.; Gauthier, J.L.; Greschner, M.; Sher, A.; Litke, A.M.; Chichilnisky, E.J. The structure of large-scale synchronized firing in primate retina. J. Neurosci. 2009, 29, 5022-5031. [CrossRef] [PubMed]

15. Schneidman, E.; Berry, M.J., II; Segev, R.; Bialek, W. Weak pairwise correlations imply strongly correlated network states in a neural population. Nature 2006, 440, 1007-1012.

16. Haken, H. Principles of Brain Functioning; Springer: Berlin, Germany, 1996.

17. Rabinovich, M.I.; Varona, P. Robust transient dynamics and brain functions. Front. Comput. Neurosci. 2011, 5, 24. [CrossRef] [PubMed]

18. Fingelkurts, A.A.; Fingelkurts, A.A. Making complexity simpler: Multivariability and metastability in the brain. Int. J. Neurosci. 2004, 114, 843-862. [CrossRef] [PubMed]

19. Jones, L.M.; Fontanini, A.; Sadacca, B.F.; Miller, P.; Katz, D.B. Natural stimuli evoke dynamic sequences of states in sensory cortical ensembles. Proc. Natl. Acad. Sci. USA 2007, 104, 18772-18777. [CrossRef] [PubMed]

20. Ito, J.; Nikolaev, A.R.; van Leeuwen, C. Dynamics of spontaneous transitions between global brain states. Hum. Brain Mapp. 2007, 28, 904-913. [CrossRef] [PubMed]

21. Kelso, J.A.S. Multistability and metastability: Understanding dynamic coordination in the brain. Philos. Trans. R. Soc. Lond. B Biol. Sci. 2012, 367, 906-918. [CrossRef] [PubMed]

22. Fingelkurts, A.A.; Fingelkurts, A.A. Operational architectonics methodology for EEG analysis: Theory and results. Neuromethods 2015, 91, 1-59.

23. Fingelkurts, A.A.; Fingelkurts, A.A.; Neves, C.F.H. Natural world physical, brain operational, and mind phenomenal space-time. Phys. Life Rev. 2010, 7, 195-249. [CrossRef] [PubMed]

24. Fingelkurts, A.A.; Fingelkurts, A.A.; Neves, C.F.H. Consciousness as a phenomenon in the operational architectonics of brain organization: Criticality and self-organization considerations. Chaos Solitons Fract. 2013, 55, 13-31. [CrossRef] 
25. Tognoli, E.; Kelso, J.A.S. Enlarging the scope: Grasping brain complexity. Front. Syst. Neurosci. 2014, 8, 122. [CrossRef] [PubMed]

26. Freeman, W.J.; Holmes, M.D. Metastability, instability, and state transition in neocortex. Neural Netw. 2005, 18, 497-504. [CrossRef] [PubMed]

27. Friston, K.J. Transients, metastability and neuronal dynamics. Neuroimage 1997, 5, 164-171. [CrossRef] [PubMed]

28. Chialvo, D.R. Emergent complex neural dynamics. Nat. Phys. 2010, 6, 744-750. [CrossRef]

29. Fingelkurts, A.A.; Fingelkurts, A.A. Mind as a nested operational architectonics of the brain. Comment on "Neuroontology, neurobiological naturalism, and consciousness: A challenge to scientific reduction and a solution" by Todd E. Feinberg. Phys. Life Rev. 2012, 9, 49-50. [CrossRef] [PubMed]

30. Afraimovich, V.; Tristan, I.; Varona, P.; Rabinovich, M. Transient dynamics in complex systems: Heteroclinic sequences with multidimensional unstable manifolds. Nonlinearity Complex. 2013, 2, 21-41. [CrossRef]

31. Freeman, W.J. Mass Action in the Nervous System; Academic Press: New York, NY, USA, 1975.

32. Freeman, W.J.; Vitiello, G. Nonlinear brain dynamics as macroscopic manifestation of underlying many-body field dynamics. Phys. Life Rev. 2006, 3, 93-118. [CrossRef]

33. Beggs, J.M.; Plenz, D. Neuronal avalanches in neocortical circuits. J. Neurosci. 2003, 23, 11167-11177. [PubMed]

34. Plenz, D. Neuronal avalanches and coherence potentials. Eur. Phys. J. Spec. Top. 2012, 205, $259-301$. [CrossRef]

35. Haken, H. Synergetics: An Introduction: Nonequilibrium Phase Transitions and Self-Organization in Physics, Chemistry and Biology; Springer: New York, NY, USA, 1983.

36. Kelso, J.A.S.; Dumas, G.; Tognoli, E. Outline of a general theory of behaviour and brain coordination. Neural Netw. 2013, 37, 120-131. [CrossRef] [PubMed]

37. Kelso, J.A.S. Behavioral neural pattern generation: The concept of neurobehavioral dynamical system (NBDS). In Cardiorespiratory and Motor Coordination; Koepchen, H.P., Huopaniemi, T., Eds.; Springer: Berlin, Germany, 1991.

38. Kelso, J.A.S.; DelColle, J.; Schöner, G. Action-perception as a pattern formation process. In Attention and Performance XIII; Jeannerod, M., Ed.; Erlbaum: Hillsdale, NJ, USA, 1990; pp. 139-169.

39. Haken, H.; Kelso, J.A.S.; Bunz, H. A theoretical model of phase transitions in human hand movements. Biol. Cybern. 1985, 51, 347-356. [CrossRef] [PubMed]

40. Bressler, S.L.; Kelso, J.A.S. Cortical coordination dynamics and cognition. Trends Cogn. Sci. 2001, 5, $26-36$. [CrossRef]

41. Fingelkurts, A.A.; Fingelkurts, A.A. Operational architectonics of the human brain biopotential field: Towards solving the mind-brain problem. Brain Mind 2001, 2, 261-296. [CrossRef]

42. Fingelkurts, A.A.; Fingelkurts, A.A. Mapping of the Brain Operational Architectonics. In Focus on Brain Mapping Research; Chen, F.J., Ed.; Nova Science Publishers, Inc.: New York, NY, USA, 2005; pp. 59-98.

43. Kaplan, A.Y. Nonstationary EEG: Methodological and experimental analysis. Uspekhi Fiziol. Nauk 1998, 29, 35-55. (In Russian)

44. Kelso, J.A.S.; Tognoli, E. Toward a complementary neuroscience: Metastable coordination dynamics of the brain. In Neurodynamics of Higher-Level Cognition and Consciousness; Kozma, R., Perlovsky, L., Eds.; Springer: Heidelberg, Germany, 2007; pp. 39-59.

45. Kelso, J.A.S.; Engstrøm, D. The Complementary Nature; MIT Press: Cambridge, MA, USA, 2006.

46. Rabinovich, M.I.; Huerta, R.; Varona, P.; Afraimovich, V.S. Transient cognitive dynamics, metastability and decision making. PLoS Comput. Biol. 2008, 4, e1000072. [CrossRef] [PubMed]

47. Werner, G. Metastability, criticality and phase transitions in brain and its models. Biosystems 2007, 90, 496-508. [CrossRef] [PubMed]

48. Schwappach, C.; Hutt, A.; Beim Graben, P. Metastable dynamics in heterogeneous neural fields. Front. Syst. Neurosci. 2015, 9, 97. [CrossRef] [PubMed]

49. Stratton, P.; Wiles, J. Global segregation of cortical activity and metastable dynamics. Front. Syst. Neurosci. 2015, 9, 119. [CrossRef] [PubMed]

50. Basar, E.; Basar-Eroglu, C.; Karakas, S.; Schurmann, M. Gamma, alpha, delta, and theta oscillations govern cognitive processes. J. Psychophysiol. 2001, 39, 241-248. [CrossRef] 
51. Freeman, W.J. Indirect biological measures of consciousness from field studies of brains as dynamical systems. Neural Netw. 2007, 20, 1021-1031. [CrossRef] [PubMed]

52. Nunez, P.L. Toward a quantitative description of large-scale neocortical dynamic function and EEG. Behav. Brain Sci. 2000, 23, 371-398. [CrossRef] [PubMed]

53. Fingelkurts, A.A.; Fingelkurts, A.A.; Neves, C.F.H. Phenomenological architecture of a mind and operational architectonics of the brain: The unified metastable continuum. New Math. Nat. Comput. 2009, 5, 221-244. [CrossRef]

54. Sengupta, B.; Tozzi, A.; Cooray, G.K.; Douglas, P.K.; Friston, K.J. Towards a neuronal Gauge Theory. PLoS Biol. 2016, 14, e1002400. [CrossRef] [PubMed]

55. Freeman, W.J. Brains create macroscopic order from microscopic disorder by neurodynamics in perception. In Disorder Versus Order in Brain Function Essays in Theoretical Neurobiology; Århem, P., Blomberg, C., Liljenström, H., Eds.; World Scientific Publishing Co.: Singapore, 2000; pp. 205-219.

56. Suntola, T. The dynamic universe-Space as a spherically closed energy system. Int. J. Astrophys. Space Sci. 2014, 2, 66-85.

57. Annila, A.; Kallio-Tamminen, T. Tangled in entanglement. Phys. Essays 2012, 25, 495-499. [CrossRef]

58. Freeman, W.J.; Kozma, R.; Vitiello, G. Adaptation of the generalized Carnot cycle to describe thermodynamics of cerebral cortex. In Proceedings of the International Joint Conference on Neural Networks (IJCNN), Brisbane, Australia, 10-15 June 2012; IEEE Press: Piscataway, NJ, USA, 2012; pp. 3229-3236.

59. Cox, D. Renewal Theory; Methuen \& Co. Ltd.: London, UK, 1962.

60. Paradisi, P.; Cesari, R.; Donateo, A.; Contini, D.; Allegrini, P. Diffusion scaling in event-driven random walks: An application to turbulence. Rep. Math. Phys. 2012, 70, 205-220. [CrossRef]

61. Paradisi, P.; Allegrini, P. Scaling law of diffusivity generated by a noisy telegraph signal with fractal intermittency. Chaos Solitons Fract. 2015, 81, 451-462. [CrossRef]

62. Allegrini, P.; Paradisi, P.; Menicucci, D.; Gemignani, A. Fractal complexity in spontaneous EEG metastable-state transitions: New vistas on integrated neural dynamics. Front. Physiol. 2010, 1, 128. [CrossRef] [PubMed]

63. Fingelkurts, A.A.; Fingelkurts, A.A.; Kaplan, A.Y. The regularities of the discrete nature of multi-variability of EEG spectral patterns. Int. J. Psychophysiol. 2003, 47, 23-41. [CrossRef]

64. Fingelkurts, A.A.; Fingelkurts, A.A. Short-term EEG spectral pattern as a single event in EEG phenomenology. Open Neuroimaging J. 2010, 4, 130-156. [CrossRef] [PubMed]

65. Lindsley, D.B. The reticular activation system and perceptual integration. In Electrical Stimulation of the Brain; Sheer, D.E., Ed.; University of Texas Press: Austin, TX, USA, 1961; pp. 331-349.

66. Jansen, B.H.; Cheng, W.-K. Structural EEG analysis: An explorative study. Int. J. Biomed. Comput. 1988, 23, 221-237. [CrossRef]

67. Sanderson, A.C.; Segen, J.; Richey, E. Hierarchical modeling of EEG signals. IEEE Trans. Pattern Anal. Mach. Intell. 1980, 2, 405-415. [CrossRef]

68. Lopes da Silva, F.N. Analysis of EEG ongoing activity: Rhythms nonstationarities. In Resent Advances in EEG and EMG Data Processing; Vamaguchi, N., Fujisawa, K., Eds.; Elsevier: Amsterdam, The Netherlands, 1981; pp. 95-115.

69. Allegrini, P.; Menicucci, D.; Bedini, R.; Fronzoni, L.; Gemignani, A.; Grigolini, P.; West, B.J.; Paradisi, P. Spontaneous brain activity as a source of ideal 1/f noise. Phys. Rev. E 2009, 80, 061914. [CrossRef] [PubMed]

70. Bianco, S.; Ignaccolo, M.; Rider, M.S.; Ross, M.J.; Winsor, P.; Grigolini, P. Brain, music, and non-poisson renewal processes. Phys. Rev. E 2007, 75, 061911. [CrossRef] [PubMed]

71. Papo, D. Timescales in cognitive neuroscience. Front. Physiol. 2013, 4, 86. [CrossRef] [PubMed]

72. Friston, K. The labile brain. I. Neuronal transients and nonlinear coupling. Philos. Trans. R. Soc. Lond. B Biol. Sci. 2000, 355, 215-236. [CrossRef] [PubMed]

73. Fingelkurts, A.A.; Fingelkurts, A.A. Alpha rhythm operational architectonics in the continuum of normal and pathological brain states: Current state of research. Int. J. Psychophysiol. 2010, 76, 93-106. [CrossRef] [PubMed]

74. Fingelkurts, A.A.; Fingelkurts, A.A.; Bagnato, S.; Boccagni, C.; Galardi, G. Toward operational architectonics of consciousness: Basic evidence from patients with severe cerebral injuries. Cogn. Process. 2012, 13, 111-131. [CrossRef] [PubMed] 
75. Fingelkurts, A.A.; Fingelkurts, A.A.; Bagnato, S.; Boccagni, C.; Galardi, G. DMN operational synchrony relates to self-consciousness: Evidence from patients in vegetative and minimally conscious states. Open Neuroimaging J. 2012, 6, 55-68. [CrossRef] [PubMed]

76. Fingelkurts, A.A.; Fingelkurts, A.A.; Bagnato, S.; Boccagni, C.; Galardi, G. Long-term (six years) clinical outcome discrimination of patients in the vegetative state could be achieved based on the operational architectonics EEG analysis: A pilot feasibility study. Open Neuroimaging J. 2016, 10, 69-79. [CrossRef] [PubMed]

77. Fingelkurts, A.A.; Fingelkurts, A.A.; Kallio-Tamminen, T. Long-term meditation training induced changes in the operational synchrony of default mode network modules during a resting state. Cogn. Process. 2016, 17, 27-37. [CrossRef] [PubMed]

78. Fingelkurts, A.A.; Fingelkurts, A.A.; Kallio-Tamminen, T. Trait lasting alteration of the brain default mode network in experienced meditators and the experiential selfhood. Self Identity 2016, 15, 381-393. [CrossRef]

(C) 2017 by the authors; licensee MDPI, Basel, Switzerland. This article is an open access article distributed under the terms and conditions of the Creative Commons Attribution (CC BY) license (http:/ / creativecommons.org/licenses/by/4.0/). 